\title{
Criminologie
}

\section{Les types de délinquantes : une étude longitudinale des causes et des conséquences}

\author{
Stephen A. Cernkovich, Catherine E. Kaukinen et Peggy C. Giordano
}

Volume 38, numéro 1, printemps 2005

Filles et déviance : perspectives développementales

URI : https://id.erudit.org/iderudit/011487ar

DOI : https://doi.org/10.7202/011487ar

Aller au sommaire du numéro

\section{Éditeur(s)}

Les Presses de l'Université de Montréal

ISSN

0316-0041 (imprimé)

1492-1367 (numérique)

Découvrir la revue

Citer cet article

Cernkovich, S. A., Kaukinen, C. E. \& Giordano, P. C. (2005). Les types de délinquantes : une étude longitudinale des causes et des conséquences. Criminologie, 38(1), 103-138. https://doi.org/10.7202/011487ar
Résumé de l'article

L'existence de différences sexuelles sur le plan du comportement antisocial est un fait largement répandu et accepté en criminologie, et ce, depuis la naissance de cette discipline. Bien que depuis une trentaine d'années les chercheurs se soient intéressés plus que jamais à la recherche théorique et empirique du comportement antisocial des femmes, force est de constater que les criminologues n'ont pas encore pleinement exploré la diversité des types de délinquance féminine. Notre examen des causes et des conséquences de la délinquance féminine s'appuie sur trois postulats de base : 1 ) la population délinquante est hétérogène 2) l'existence de types distincts de délinquantes est le produit de processus causaux qui sont à la fois communs et distincts et 3) les conséquences à long terme de la délinquance varient selon le type de délinquantes. Les données autorapportées ont été recueillies à partir d'un échantillon de répondantes interrogées en 1982, alors qu'elles étaient adolescentes, et subséquemment en 1992, alors qu'elles avaient atteint l'âge adulte. Nos analyses ont décelé des facteurs étiologiques uniques et communs à l'ensemble des types de délinquantes, ainsi que des événements de vie variant en fonction d'une diversité de dimensions comportementales, personnelles et interpersonnelles. Nos résultats font ressortir qu'une consommation de drogues durant l'adolescence a des effets particulièrement délétères chez les femmes lors du passage de l'adolescence à l'âge adulte.
Tous droits réservés @ Les Presses de l'Université de Montréal, 2005
Ce document est protégé par la loi sur le droit d'auteur. L’utilisation des services d'Érudit (y compris la reproduction) est assujettie à sa politique d'utilisation que vous pouvez consulter en ligne.

https://apropos.erudit.org/fr/usagers/politique-dutilisation/ 


\title{
Les types de délinquantes: une étude longitudinale des causes et des conséquences ${ }^{1}$
}

\author{
Stephen A. Cernkovich \\ Département de sociologie \\ Bowling Green State University \\ scernco@bgnet.bgsu.edu \\ Catherine E. Kaukinen \\ Département de criminologie et de justice criminelle \\ University of South Carolina \\ kaukinen@gwm.sc.edu
}

\section{Peggy C. Giordano \\ Département de sociologie \\ Bowling Green State University \\ pgiorda@bgnet.bgsu.edu}

RÉSUMÉ • L'existence de différences sexuelles sur le plan du comportement antisocial est un fait largement répandu et accepté en criminologie, et ce, depuis la naissance de cette discipline. Bien que depuis une trentaine d'années les chercheurs se soient intéressés plus que jamais à la recherche théorique et empirique du comportement antisocial des femmes, force est de constater que les criminologues n'ont pas encore pleinement exploré la diversité des types de délinquance féminine. Notre examen des causes et des conséquences de la délinquance féminine s'appuie sur trois postulats de base: 1) la population délinquante est hétérogène 2) l'existence de types distincts de délinquantes est le produit de processus causaux qui sont à la fois communs et distincts et 3) les conséquences à long terme de la délinquance varient selon le type de délinquantes. Les données autorapportées ont été recueillies à partir d'un échantillon de

1. Cette étude a été financée par les subventions de recherche $\mathrm{n}^{\text {os }} \mathrm{MH} 29095$ et $\mathrm{MH}$ 46410 du Public Health Service, par le National Institute of Mental Health et par une subvention du Research Challenge Program octroyée par le Obio Board of Regents. Texte traduit par C. Pilon et révisé par N. Lanctôt. 
répondantes interrogées en 1982, alors qu'elles étaient adolescentes, et subséquemment en 1992, alors qu'elles avaient atteint l'âge adulte. Nos analyses ont décelé des facteurs étiologiques uniques et communs à l'ensemble des types de délinquantes, ainsi que des événements de vie variant en fonction d'une diversité de dimensions comportementales, personnelles et interpersonnelles. Nos résultats font ressortir qu'une consommation de drogues durant l'adolescence a des effets particulièrement délétères chez les femmes lors du passage de l'adolescence à l'âge adulte.

RÉSUMÉ - The gender difference in antisocial behaviour has been an accepted fact in criminology since the birth of the discipline. Although the past thirty years have seen more theorizing and research on antisocial behaviour among females than at any previous time, criminologists have yet to fully explore the diversity of types that make up the female offender population. Our examination of the causes and consequences of female offending begins with three basic assumptions: 1) a heterogeneity of types characterizes the offending population 2) distinct offender types are the product of both shared and unique causal processes and 3) the longterm consequences of offending vary among offender types. Self-report data were gathered from a sample of female respondents interviewed in 1982 when they were adolescents, and subsequently in 1992 when they were young adults. Our analyses identify both common and unique etiologies among several distinct types of offenders, as well as differential life course outcomes along a variety of behavioural, personal and interpersonal dimensions. Our findings are noteworthy in suggesting that adolescent drug use has particularly deleterious effects for females as they transition to young adulthood.

Pour une variété de bonnes raisons, les criminologues se sont longtemps intéressés aux délinquants masculins, délaissant du coup les nondélinquantes et les délinquantes. Bien qu'aucun de ces deux groupes n'ait été complètement oublié par les chercheurs, il reste que c'est principalement depuis la fin des années 1970 que la recherche théorique et empirique sur la délinquance féminine a pris son envol. Par contre, les non-délinquants et les délinquantes ont surtout été traités comme des points de comparaison, les théories et les recherches mettant d'abord en valeur les délinquants masculins. À la lumière du savoir développé dans le domaine de la délinquance masculine, il est évident que nous avons tout à gagner à en apprendre davantage sur les non-délinquants et sur les délinquantes. Cette recherche vise donc à parfaire nos connaissances dans ces champs de savoir en procédant à un examen détaillé des types d'adolescentes conformistes et délinquantes. Nous nous intéresserons particulièrement aux causes et aux conséquences de la délinquance féminine et à leurs variations en fonction des différences qualitatives propres à chaque type de délinquante. 
Chez les filles et les garçons, les conduites délinquantes constituent la norme durant l'adolescence (Elliott et al., 1985 ; Moffitt, 1993 ; Moffitt et al., 1996; Lanctôt et Le Blanc, 2002). Bien que la plupart des grandes théories criminologiques abordent, explicitement ou implicitement, les raisons pour lesquelles certains adolescents résistent aux facteurs qui conduisent une majorité de leurs pairs à commettre des actes délinquants, très peu d'entre elles se sont appuyées sur des recherches ou des hypothèses essentiellement axées sur les adolescents dits conformistes. Qui plus est, ces non-délinquants n’ont pas non plus été perçus et étudiés comme un type comportemental distinct et qualitativement différent dans les typologies ayant jusqu'ici été élaborées. Cela dit, une constatation s'impose: les adolescents conformistes comptent davantage de filles que de garçons. Il est bien connu que les hommes sont considérablement plus antisociaux que les femmes et que l'implication de ces dernières dans la délinquance est intrinsèquement moins sérieuse et moins persistante (Cernkovich et Giordano, 1979; McCord, 1993; Steffensmeier et Streifel, 1993 ; Lanctôt et Le Blanc, 2002). Ainsi, dans une récente étude longitudinale sur les trajectoires délinquantes, Chung, Hill, Hawkins, Gilchrist et Nagin (2002) démontrent que si la délinquance chronique est plus élevée chez les adolescents que chez les adolescentes $(9,9 \%$ vs $4,1 \%)$, la non-délinquance, elle, est très fréquente chez ces dernières (32,2\% vs $16 \%)$.

Ces références aux différences sexuelles sont largement répandues et acceptées dans le domaine de la criminologie. Selon les premières explications sur le sujet, la délinquance féminine serait le fait de jeunes filles anormales et souffrant de pathologies personnelles diverses (Lombroso et Ferrero, 1916; Barker et Adams, 1962; Reige, 1972; Robins, 1986). Cette conception de la délinquance féminine a persisté durant plusieurs décennies et c'est seulement à partir des années 1970 que la multiplication des recherches sur le sujet a démontré que les femmes manifestaient également de façon indéniable des comportements antisociaux. Un nouveau type de délinquante venait ainsi d'être mis au jour: une délinquante au comportement plus agressif, plus violent ou, à tout le moins, plus masculin que celui qu'on ne le lui conférait traditionnellement (Adler, 1975; Simon, 1975; Ageton, 1983; Berger, 1989). Plus encore, cette nouvelle vision soutient que la délinquance féminine résulte de pressions sociales et culturelles, plutôt que d'influences personnelles pathologiques. Durant cette même période, il appert que si les femmes commettent davantage de crimes que par le passé, leurs 
actes de délinquance demeurent tout de même moins sérieux et moins diversifiés que ceux de leurs homologues masculins (Steffensmeier, 1980 ; Steffensmeier et Allen, 1988). Quelle que soit la façon dont les délinquantes sont perçues, il semble évident qu'à partir des années 1970, un nombre grandissant de chercheurs ont inclu la gent féminine dans leurs échantillons et ont étudié la délinquance et la criminalité chez les femmes plus diligemment que jamais auparavant (Cernkovich et Giordano, 1979; Giordano et Cernkovich, 1979; Campbell, 1981; Norland et al., 1981; Silverthorn et Frick, 1999; Côté et al., 2001; Silverthorn et al., 2001; D'Unger et al., 2002; Lanctôt et Le Blanc, 2002). Dès lors, nous en connaissons considérablement plus aujourd'hui sur la délinquance féminine qu'il y a trente ans.

Reconnaissant l'importance de certaines méthodes d'analyse l'étiologie de la délinquance féminine en regard de la délinquance masculine ou encore le développement de théories spécifiques à chaque sexe (voir Lanctôt et Le Blanc, 2002) - la présente recherche s'intéressera à une problématique en particulier: les types de délinquantes. Nous croyons que les visions stéréotypées de la délinquance des femmes qui ont prévalu historiquement nous ont empêchés de voir qu'il existait en fait divers types de délinquantes. En effet, les délinquantes ont longtemps été perçues comme un groupe relativement homogène. D'emblée, force est de constater que certains délits tels que la prostitution et les crimes de violence familiale ont été associés à la délinquance féminine. Il en est de même pour les délits résultant d'une stratégie de survie, en réponse à la violence physique ou sexuelle sévissant dans le milieu familial. Quoi qu'il en soit, alors que beaucoup d'efforts ont été déployés pour établir des typologies de délinquants masculins (Clinard et Quinney, 1971 ; Farrington, 1973 ; 1977 ; Gibbons, 1985 ; Moffitt, 1993 ; Nagin et Land, 1993 ; D’Unger et al., 1998), c'est plutôt le contraire du côté des délinquantes.

Parallèlement, nombre de recherches récentes ont porté sur les trajectoires des adolescentes (Dunforth et Elliott, 1984 ; Silverthorn et Frick, 1999; Côté et al., 2001; Silverthorn et al., 2001; D'Unger et al., 2002). La plupart de ces recherches indiquent que les trajectoires délinquantes des adolescents et des adolescentes sont très semblables, autant dans leurs formes que par leurs facteurs étiologiques, quoique les délinquantes tendent à s'impliquer dans des activités antisociales moins graves et moins persistantes que leurs homologues masculins (Lanctôt et Le Blanc, 2002). Bien que toutes ces recherches aient fourni une 
masse importante de nouvelles informations à propos de la délinquance féminine, il convient de mentionner qu'elles se sont principalement concentrées sur les conduites propres à chaque trajectoire, plutôt que sur les caractéristiques des délinquantes de ces trajectoires. Une importante exception concerne le travail de Lanctôt et Le Blanc (2000) sur les caractéristiques personnelles et sociales de trois types de trajectoires déviantes chez des adolescentes judiciarisées: les trajectoires déviantes bénignes, déclinantes et persistantes. Quoi qu'il en soit, cette étude s'est uniquement intéressée à la période de l'adolescence, de sorte que les chercheurs n'ont pas été en mesure d'évaluer les conséquences sur la vie d'adulte de ces comportements déviants précoces (Lanctôt et Le Blanc, 2002: 137-138).

Sliverthorn et Frick (1999), ainsi que Sliverthorn, Frick et Reynolds (2001) se sont également attardés aux différences qualitatives entre les types de délinquantes. Ils ont examiné si les deux types dominants de délinquants masculins dégagés par Moffitt (1993) - les délinquants persistants et les individus dont la délinquance se limite à l'adolescence - s'appliquaient également aux délinquantes. Silverthorn et Frick (1999) ont remis en question cette explication de la délinquance qui ne repose que sur deux trajectoires. Ils ont plutôt proposé l'ajout d'une troisième trajectoire propre aux délinquantes: la trajectoire tardive (delayed-onset pathway). Contrairement aux garçons, les filles qui ont des troubles de conduite précoces persistent peu dans les conduites antisociales à l'âge adulte. Durant l'enfance, divers facteurs sociaux et biologiques contribueraient à supprimer le comportement antisocial chez la plupart des filles, incluant celles qui sont aux prises avec des troubles de conduite. Ce n'est que lorsque se manifestent les changements biologiques et sociaux associés à la puberté que les filles les plus vulnérables commencent à adopter des comportements délinquants (Sliverthorn et al., 2001 : 172). En résumé, Sliverthorn et Frick (1999: 113-114) soutiennent qu'il n'existe qu'une seule trajectoire développementale pour les adolescentes antisociales: la trajectoire tardive. Bien que cette trajectoire typiquement féminine soit très semblable à la trajectoire des délinquants persistants, qui débute dès l'enfance, il reste que chez les filles les comportements délinquants n'apparaissent qu'à l'adolescence. À l'instar des résultats observés pour les délinquants précoces et persistants, Silverthorn et Frick (1999: 116-21) affirment que les filles qui s'engagent tardivement dans la délinquance présentent des déficits cognitifs et neuropsychologiques, ont une faible estime d'elles-mêmes et vivent dans des 
environnements familiaux peu favorables à leur développement. Plus encore, Sliverthorn, Frick et Reynolds (2001) ont remarqué que ces jeunes filles connaissaient bien souvent une adolescence et une vie d'adulte difficiles à plusieurs égards: taux élevés d'arrestations et d'activités illégales autorévélées, troubles afférents à la personnalité antisociale, abus de substances psychotropes, grossesse à l'adolescence, décrochage scolaire, tentatives de suicide, faible contrôle de soi, relations interpersonnelles marquées par l'insensibilité et le manque d'émotions, ainsi qu'une variété de troubles intériorisés.

Dans la même veine, Côté et al. (2001) ont observé que chez les filles, les troubles de la conduite constituent de bons prédicteurs de problèmes divers, dont l'alcoolisme et la toxicomanie, les troubles intériorisés, les troubles afférents à la personnalité antisociale, la mort précoce et violente, les arrestations criminelles, un faible niveau de scolarité et un état de santé précaire. De plus, Côté et al. (2001) définissent quatre trajectoires de délinquance chez les adolescentes. Au même titre que les études précédentes dans ce domaine, leurs recherches se concentrent surtout sur les différences comportementales entre les trajectoires et sur leur capacité de prédire les comportements futurs à partir des comportements manifestés antérieurement. Au-delà de leur volonté d'évaluer la proportion de filles de chaque trajectoire aux prises avec des troubles de conduite, le champ de leur recherche ne s'étend pas à l'exploration des distinctions qualitatives entre les groupes de délinquantes.

De leur côté, en analysant une cohorte née à Philadelphie (Tracy et al., 1990), D'Unger et al. (2002) évaluent si les délinquantes forment un groupe aussi hétérogène que les délinquants. Si les résultats de l'équipe de D’Unger et al.(1998) avaient précédemment permis de reconnaitre cinq catégories de délinquants, les résultats de leur plus récente étude (D’Unger et al., 2002) n'établissent, en revanche, que trois catégories distinctes de délinquantes: les non-délinquantes, les délinquantes à faible fréquence d'actes criminels et les délinquantes à fréquence élevée d'actes criminels. Mais au-delà de cette classification, il nous faut préciser que ces chercheurs se sont surtout intéressés aux formes que pouvait revêtir la courbe âge-crime chez les filles, plutôt qu'aux facteurs étiologiques et aux conséquences associés à chaque type de délinquante. Autrement dit, l'orientation de leurs travaux n'a pas tant porté sur les caractériques des types de délinquantes que sur l'âge de ces dernières aux étapes du début, de l'apogée et du désistement de leurs activités délinquantes. Si on leur reconnaît une importante contribution 
sur le plan des comparaisons entre les sexes, cela demeure beaucoup moins vrai en ce qui concerne les distinctions qualitatives des types de délinquantes (2002).

L'objectif de la présente recherche est d'accroître les connaissances sur les trajectoires délinquantes des adolescentes, en se concentrant particulièrement sur les types qualitativement distincts de délinquantes. Ce faisant, nous combinons deux traditions de longue date en criminologie: la nécessité de distinguer les délinquants des non-délinquants et l'importance de discerner des types qualitativement différents de délinquants. Alors que certains chercheurs qui étudient les carrières criminelles ou les trajectoires développementales se sont attardés aux processus ou à la séquence événementielle de la délinquance (Dunforth et Elliott, 1984 ; Gottfredson et Hirschi, 1986; Blumstein et al., 1988; Loeber et Le Blanc, 1990; Moffitt, 1993; Patterson et Yoerger, 1993; Sampson et Laub, 1997; D’Unger et al., 1998; Nagin et Sampson, 1998; Silverthorn et Frick, 1999; Côté et al., 2001; Silverthorn et al., 2001; D'Unger et al., 2002), notre intérêt réside plus simplement dans la différenciation des types de délinquantes. Selon Sampson and Laub (2003), l'importance d'établir des types distincts de délinquants est reconnue par plusieurs criminologues spécialisés dans l'étude du développement de la délinquance (Loeber et LeBlanc, 1990; Moffitt, 1993 ; Patterson et Yoerger, 1993), ce qui suggère qu'une étiologie distincte caractérise chaque type de délinquants. Il demeure néanmoins que, bien que plusieurs chercheurs aient étudié les types de délinquants et/ou les trajectoires individuelles menant à diverses carrières criminelles, leur intérêt a toujours porté sur les délinquants chroniques, par opposition aux délinquants non chroniques, ou sur les délinquants précoces et persistants, par opposition à ceux qui affichent une délinquance limitée à l'adolescence (Moffitt, 1993). Ces recherches ont également bien souvent ignoré la délinquance des adolescentes ou, du moins, en ont rarement tenu compte (Blumstein et al., 1988; Moffitt, 1993 ; Sampson et Laub, 1993; Loeber, 1996; Cernkovich et Giordano, 2001 ; Chung et al., 2002). Dès lors, nous désirons accorder aux femmes, en contrepartie, toute l'attention qu'elles méritent en établissant des différences qualitatives existant entre les types de délinquantes, exercice qui s'appuiera sur l'examen des causes et des conséquences de leurs comportements.

Nous souscrivons à la théorie de Chung et al. (2002) qui stipule que l'évidente hétérogénéité de la population délinquante suggère que les 
variables causales associées à la délinquance different d'un type de délinquant à un autre. Bien qu'une série de variables semblables puissent être associées à plus d'un type de délinquance, voire même à tous les types, il demeure que ces variables jouent un rôle causal différent selon le type de délinquance, ou encore n'interviennent que dans des circonstances uniques. À cet effet, plusieurs recherches ont relevé des ressemblances entre les prédicteurs des différents types de délinquants (Patterson et Yoerger, 1993, Fergusson et al., 1996; Patterson, 1996; Patterson et Yoerger, 1997; Fergusson et al., 2000), ainsi que l'existence de prédicteurs uniques à chaque type (Farrington et Hawkins, 1991; Loeber et al., 1991; Patterson et Yoerger, 1997; Rutter et al., 1998). Malheureusement, comme peu de recherches sont basées sur des échantillons féminins, il est difficile de savoir si les résultats de ces travaux s'appliquent également aux délinquantes. Par exemple, en utilisant un échantillon composé à $49 \%$ de filles, Chung et ses collègues (2002) n'ont pas évalué leurs modèles d'une façon distincte pour chaque sexe. Ils se sont plutôt limités à vérifier si la répartition des sexes à travers les différentes trajectoires délinquantes se distinguait. Cette décision d'inclure le sexe comme variable contrôle est tout à fait cohérente avec nombre de recherches antérieures sur le sujet. Néanmoins, l'effet cumulatif de ces recherches, qui ont ignoré les différences entre les sexes ou qui n'ont inclus le sexe qu'à titre de variable contrôle, démontre que la délinquance féminine n'a reçu qu'un intérêt accessoire et que, en comparaison avec la délinquance des garçons, nous n'en savons que très peu sur l'hétérogénéité des types de délinquantes.

\section{Cadre théorique}

Notre examen des causes et des conséquences de la délinquance féminine repose sur trois postulats de base : 1) la population délinquante est hétérogène 2) l'existence de types distincts de délinquantes est le produit de processus causaux qui sont à la fois communs et distincts et 3) les conséquences à long terme de la délinquance varient selon le type de délinquantes. En examinant les causes des patrons uniques de délinquance chez les adolescentes, nous nous appuyons sur deux perspectives théoriques: celle de la régulation sociale et celle de l'apprentissage social. Chacune de ces théories met en évidence l'incidence de la famille, des pairs et de l'école sur les comportements des adolescents - 
trois domaines d'une importance capitale et indéniable, selon la documentation générale sur la délinquance. La théorie de la régulation sociale (Hirschi, 1969) soutient que les individus évitent d'adopter des comportements délinquants lorsqu'ils sont attachés à la société dans laquelle ils vivent. En effet, les individus qui sont attachés à leurs parents et à leurs amis, qui s'investissent dans leur cheminement scolaire, qui participent à des activités conventionnelles et qui intériorisent des valeurs prosociales sont beaucoup moins susceptibles de s'engager dans la délinquance. De son côté, si la théorie de l'apprentissage social reconnait l'importance des influences prosociales de la famille et de l'école, elle accorde une attention toute particulière à l'influence des pairs. Selon cette perspective, les individus qui adoptent des comportements délinquants le font parce que ces comportements ont été montrés, encouragés et renforcés par leurs groupes de pairs. Bref, les individus apprennent à recourir à certaines conduites, qu'elles soient prosociales ou antisociales, parce qu'elles ont été renforcées dans le passé. Ces individus escomptent ensuite que ces conduites continueront d'être renforcées à l'avenir.

En procédant à l'examen des conséquences de la délinquance, nous nous appuyons sur le modèle de dépendance. Selon ce modèle, le comportement délinquant a des conséquences certaines sur le comportement futur, ceci à la faveur d'une variété d'influences médiatrices. En effet, l'adoption d'un comportement antisocial change la vie d'un délinquant d'une multitude de façons et ce sont précisément ces changements qui, à leur tour, influent sur les divers événements qui ponctuent la vie d'un délinquant. L'hypothèse la plus fondamentale issue de cette perspective est que le comportement d'un individu a des incidences causales sur la probabilité de ses actes ultérieurs (Nagin et Paternoster, 2000). Plus encore, il semble que l'adoption par un individu d'un comportement antisocial entraîne des conséquences négatives sur sa vie, particulièrement en ce qui concerne les relations avec la famille, les amis et les professeurs. Ainsi, l'adoption d'un comportement antisocial mène souvent à un effritement de ces relations, lequel ajoute aux risques qu'un individu ne commette de futurs crimes. Qui plus est, l'adoption de comportements antisociaux mène souvent à une intensification des rapports avec des pairs délinquants. Conséquemment, les motivations à commettre davantage de délits s'en trouvent intensifiées par les pressions, les encouragements et le soutien des pairs délinquants (Nagin et Paternoster, 2000). 
En nous appuyant sur la logique du modèle de dépendance, nous avançons que les différents types de délinquance entraînent différentes conséquences dans la vie des individus. Par exemple, les conséquences d'une délinquance grave à l'adolescence seront passablement différentes de celles d'une délinquance essentiellement liée à la consommation de drogues. À cet effet, plusieurs raisons portent à croire que l'usage de drogues chez les adolescentes est particulièrement lourd de conséquences. Wallace et al. (2003) ont remarqué d'importants changements au fil du temps sur le plan de la consommation de drogues chez les filles. En effet, il semble que les modèles de consommation des adolescentes ressemblent de plus en plus à ceux des garçons. À la lumière de ces tendances, les auteurs proposent d'évaluer si les facteurs de risque et de protection recensés dans les recherches antérieures se transposent aux adolescentes. De plus, puisque la consommation de drogues a d'importantes répercussions sur le développement et la stabilité des relations interpersonnelles des adolescentes, il est important d'examiner ces conséquences sociales et comportementales au moment de la transition vers l'âge adulte. Enfin, puisque la consommation de drogues à l'adolescence augmente les risques d'une telle consommation à l'âge adulte (Chassin et al., 2002) et que l'abus de substances psychotropes est associé à de graves dysfonctions sociales chez les filles (Laukkanen et al., 2001), il est primordial d'étudier attentivement les répercussions de cette conduite.

Au-delà de l'importance que revêtent de telles problématiques, les recherches antérieures n'ont guère exploré la façon dont les conséquences peuvent différer selon le type de délinquance des adolescentes, par exemple entre une délinquante qui s'adonne à une délinquance grave et une délinquante dont les activités sont surtout liées à la drogue. Comme les informations colligées sur le sujet ne nous permettent pas d'énoncer d'hypothèses précises, nous nous proposons d'étudier le lien entre la diversité des conséquences liées à des comportements antisociaux durant l'adolescence (ex. : perpétration d'actes criminels, consommation abusive d'alcool et de drogues) et les types de délinquantes.

\section{Méthodologie}

Les données utilisées dans le cadre de cette étude proviennent d'un échantillon de 942 répondants résidant dans des maisons privées de la région métropolitaine de Toledo, en Ohio. Les répondants ont d'abord 
été interviewés en 1982 (temps 1), alors qu'ils étaient adolescents, puis une deuxième fois en 1992 (temps 2), alors qu'ils étaient adultes. En 1982, une technique modifiée d'échantillonnage multiéchelle a permis de sélectionner aléatoirement des secteurs de cette région métropolitaine. Parmi les répondants interviewés au temps 1 de l'étude, $51 \%$ sont de sexe féminin et $45 \%$ sont des Blancs. Ceux qui ne sont pas de race blanche sont majoritairement des Afro-américains, lesquels représentent $50 \%$ de l'échantillon. L'âge des répondants varie entre 12 et 19 ans. Le taux de participation au temps 2 de l'étude est de $77 \%$ du nombre initial de participants, compte tenu du décès confirmé de 10 participants. Des 721 répondants interviewés au temps 2, 55\% sont des femmes et $47 \%$ sont de race blanche. Chez les non-blancs, la proportion d'Afroaméricains atteint $95 \%$. L'âge des sujets varie entre 22 et 29 ans, la moyenne étant de 25,31 ans. Le revenu moyen est de 21100 \$ par ménage et, au moment de la deuxième entrevue, $30 \%$ des répondants n'occupaient pas d'emploi.

Plus précisément, l'échantillon de la présente étude est composé de toutes les femmes interrogées au temps $1(\mathrm{n}=484)$ et au temps 2 $(\mathrm{n}=398)$. L'âge moyen des participantes est de 15,39 ans et varie entre 12 et 19 ans pour le temps 1, alors qu'il est de 25,39 ans et varie entre 22 et 29 ans pour le temps 2 . Au temps 1, $45 \%$ des femmes interviewées sont de race blanche, par rapport à $49 \%$ d'Afro-américaines et au temps 2, ces proportions sont respectivement de $48 \%$ et de $47 \%$. Le revenu moyen par ménage de ces femmes est de 23389 \$ au temps 2 et $36 \%$ d'entre elles affirmaient être sans emploi au moment de l'entrevue.

\section{Identification et définition des types de délinquantes: une analyse de structure latente}

Pour en arriver à conceptualiser la délinquance comme une variable normalement distribuée dans la population, la plupart des recherches antérieures se sont appuyées sur des échelles cumulées ainsi que sur des modèles d'analyse factorielle. De leur côté, Brownfield et Sorenson (1987) affirment qu'il est tout aussi valable de conceptualiser la délinquance comme une variable qualitative définie à l'aide de plusieurs catégories mutuellement exclusives. En s'inspirant de cette logique, notre démarche s'appuie sur l'analyse de structure latente (ASL) pour définir empiriquement le construit de la délinquance à partir d'un 
ensemble de comportements criminels et statutaires ${ }^{2}$. Au lieu de présumer de la structure que revêt la délinquance pour l'ensemble de l'échantillon, l'analyse de structure latente dégage, au moyen de critères empiriques, des formes distinctes de délinquance.

À partir d'une classification croisée des réponses aux items de délinquance, six variables ont été utilisées afin de représenter la structure latente de la délinquance à l'adolescence. Les six variables utilisées dans l'ASL proviennent des réponses dichotomiques se rapportant aux catégories suivantes, mesurées au temps 1:1) délits graves et violents (agression sexuelle, vol qualifié, bataille de gangs, agression d'une personne, port d'une arme) 2) délits mineurs violents (frapper quelqu'un ou lancer un objet à quelqu'un) 3) délits graves contre la propriété (vol de voiture, vol d'envergure, introduction avec effraction) 4) délits mineurs contre la propriété (achat ou recel de biens volés, vol de biens d'une valeur de 5 à $50 \$$, menus larcins ou dommages à la propriété) 5) vente de drogues (marijuana ou drogues dures) et 6) consommation de drogues (avoir consommé des drogues dans le but de "se défoncer»). De ces six variables dichotomiques découlent logiquement 64 catégories possibles de délinquance. L'objectif de l'ASL est de déterminer le nombre de catégories qui représente le mieux la variabilité des données présentes dans le tableau croisé (6 X 6). Les résultats indiquent que le modèle qui est le plus approprié à nos données est formé de quatre classes.

L'analyse rapporte d'abord un groupe d'adolescentes qualifiées de conformistes. Cette catégorie contient $22 \%$ des répondantes de l'échan-

2. L'analyse de structure latente (ASL) est souvent décrite comme une analyse factorielle conçue pour les variables catégorielles qui permet d'utiliser des variables directement observées pour construire des concepts théoriques et des types multidimensionnels, tels que la délinquance et la criminalité (Heinen, 1996). L'ASL permet également de découvrir un ensemble de comportements délinquants mutuellement exclusifs qui tiennent compte de la distribution des cas à l'intérieur d'un tableau croisé d'items auto-rapportés par les répondants (McCutcheon, 1987 : 8). L'ASL permet d'établir des types de délinquants basés sur les réponses obtenues à un certain nombre de questions individuelles portant sur la délinquance. Dans le cadre de cette recherche, nous avons analysé le modèle de structure latente en utilisant le programme Maximum likelibood latent structure analysis (MLLSA) développé par Clifford Clogg dans le cadre du Categorical data analysis system (CDAS). Cette analyse peut être consultée avec l'accord préalable des auteurs. Les estimations de paramètres fournies par le programme MLLSA permettent une description narrative des catégories de délinquants à partir de la variable latente. Les données issues de la MLLSA incluent le nombre de répondants pour chaque catégorie de délinquants, ainsi que la probabilité d'une réponse positive pour chacune des questions individuelles portant sur la délinquance. L'annexe 1 expose les résultats de notre ASL. 
tillon qui affirment ne jamais avoir participé à une quelconque activité délinquante. Le plus important groupe d'adolescentes répertorié par l'analyse de structure latente est celui des délinquantes ayant commis des délits mineurs avec violence. En effet, cette catégorie de délinquantes représente $51 \%$ des adolescentes de l'échantillon. La quasi-totalité (99\%) de ces délinquantes ont commis des actes d'une violence mineure, par exemple frapper les autres ou leur lancer des objets. Plusieurs des adolescentes de ce groupe ont également commis des délits contre la propriété. Il importe de souligner qu'aucune de ces adolescentes n'était impliquée dans la vente ou dans la consommation de drogues. Notre intérêt pour les délinquantes de cette catégorie s'explique par le caractère normatif de leurs conduites. De façon très concrète, il se pourrait que la différence la plus significative entre les types de délinquantes ne se situe pas tant entre les adolescentes conformistes et les rares délinquantes qui s'adonnent à une délinquance sérieuse, qu'entre les adolescentes conformistes et les nombreuses adolescentes qui posent des actes de délinquance mineure avec violence. Notre analyse veillera à distinguer ces deux derniers groupes de délinquantes et à cerner les différences qui opposent celles-ci aux adolescentes impliquées dans des délits plus graves. La troisième catégorie de délinquantes concerne principalement les consommatrices de drogues. À l'intérieur de ce plus petit groupe - $16 \%$ de l'échantillon - la participation à des activités délinquantes est principalement limitée à la consommation et à la vente de drogues. Ces adolescentes sont également compromises dans quelques délits contre la propriété et dans quelques délits mineurs avec violence. La dernière catégorie concerne les adolescentes se livrant à des formes plus graves de délits avec violence et contre la propriété. Ces délinquantes sérieuses, qui s'impliquent dans une délinquance grave, représentent $11 \%$ de l'échantillon. La presque totalité $(90 \%)$ des adolescentes comprises dans ce groupe ont affirmé avoir commis un crime grave avec violence. Mentionnons enfin que ces délinquantes sont également très actives dans la consommation et la vente de drogues, ainsi que dans des délits contre la propriété.

\section{Les conséquences de la délinquance}

À l'exception de la participation à des actes de délinquance durant l'adolescence, chacune des variables mentionnées ci-dessous a été mesurée lors du temps 2 de l'étude, soit au moment où les répondantes étaient de jeunes adultes. 
Comportements criminels à l'âge adulte (alpha $=0,839)$ - Cette échelle a été mesurée à partir d'une version modifiée des échelles de délinquance autorapportée mises au point par Elliott et Ageton (1980). Ces échelles indiquent le niveau autorévélé d'implication dans des délits contre la propriété, contre la personne et dans ceux liés à la drogue et à l'alcool, le tout au cours de la dernière année. Les réponses s'étalent sur une échelle de 1 (jamais) à 9 (plus d'une fois par jour). Chacun des items de cette échelle a reçu un indice pondéré de gravité variant de 1,42 (consommation de drogues) à 25,85 (agression sexuelle), indice tiré du National Survey of Crime Severity (Wolfgang et al., 1985; Cernkovich et Giordano, 1992). Les résultats obtenus pour les comportements criminels à l'âge adulte représentent le score moyen de la somme des produits entre la fréquence de chaque item et son indice pondéré de gravité ${ }^{3}$.

Comportements délinquants (alpha $=0.843)$ - Cette échelle a été mesurée au temps 1 à partir des délits statutaires, des délits contre la propriété et des délits violents rapportés par les répondantes au cours des 12 derniers mois. Au même titre que l'échelle des comportements criminels à l'âge adulte, cette échelle se rapporte à la version modifiée de la délinquance autorévélée (Elliott et Ageton, 1980). Les items de l'échelle ont été pondérés et le résultat total a été calculé de la manière décrite cihaut ${ }^{4}$.

3. L'échelle des comportements criminels à l'âge adulte comprend les 19 items suivants: endommager ou détruire la propriété, voler un véhicule à moteur, voler un objet d'une valeur de plus de $50 \$$, transporter une arme dissimulée, voler un objet d'une valeur de $5 \$$ et moins, attaquer quelqu'un avec l'intention de le blesser sérieusement, participer à une bagarre de gangs, vendre de la marijuana ou du haschisch, frapper quelqu'un, vendre des drogues dures, avoir eu des relations sexuelles avec quelqu'un sans son consentement, être en état d'ébriété sur la voie publique, s'introduire par effraction, consommer des drogues dans le but de «se défoncer», frauder le service des impôts, voler des objets de petite valeur au travail, voler des objets d'une valeur de plus de $50 \$$ au travail, utiliser des cartes de crédit sans l'autorisation du détenteur ou encaisser un faux chèque et commettre des fraudes. Chacun de ces items a été pondéré afin d'éviter l'effet déformant de la faible fréquence des comportements graves dans le calcul du score total de participation à des activités criminelles. Cette stratégie concorde avec le commentaire de Moffitt (1997: 46) selon lequel les recherches qui négligent de prendre en considération l'hétérogénéité des comportements antisociaux ont laissé dans l'ombre le rôle de certains facteurs causals et sous-estimé l'influence des autres. De telles sous-estimations sont particulièrement fréquentes pour les échantillons qui comportent un faible nombre de délinquants persistants et de criminels graves. Cela est encore plus vrai lorsque l'on considère que les données relatives aux comportements antisociaux que fournissent habituellement ces échantillons sont déformées par les rapports obtenus d'un nombre beaucoup plus grand de personnes dont la délinquance était jugée moins grave.

4. Les 27 items qui composent l'échelle des comportements délinquants sont: le vandalisme, le vol de véhicules à moteur, le vol d'objets d'une valeur supérieure à 50 \$, l'achat, la 
Abus de drogues et d'alcool à l'âge adulte (alpha $=0,925)$ - Cette échelle indique jusqu'à quel point la consommation de drogues et d'alcool affecte la santé, le travail, les relations interpersonnelles et les problèmes de comportement de la répondante et/ou des gens avec qui elle interagit. Les répondantes indiquaient à quelle fréquence - sur une échelle de 1 (jamais) à 9 (plus d'une fois par jour) - leur consommation de drogues/alcool avait entrainé les conséquences suivantes: 1) se sentir plus ou moins bien le lendemain 2) se sentir incapable d'accomplir adéquatement son travail ou d'assister à ses cours 3) s'être mise dans le pétrin vis-à-vis de sa famille ou de ses amis 4) avoir frappé un membre de sa famille 5) s'être bagarrée avec d'autres 6) avoir volé de l'argent ou des biens dans le but de se procurer de l'alcool ou de la drogue. L'obtention d'un résultat élevé sur cette échelle indique un niveau élevé de problèmes de consommation d'alcool et/ou de drogues.

Identité sociale (alpha $=0,524)$ - Des questions révisées, inspirées du National Youth Survey (Matsueda, 1992), ont été utilisées pour savoir comment les répondantes pensaient que les gens de leur entourage les percevaient. Les répondantes indiquaient à quel point elles pensaient que les gens de leur entourage les décriraient comme suit: 1) un fauteur de troubles 2) un enfant modèle 3) d'humeur changeante 4) un enfant agité, dissipé 5) studieux 6) ambitieux 7) empressé de plaire aux autres 8) aimé des gens 9) ayant besoin de consulter un spécialiste 10) quelqu'un qui tombe sur les nerfs des gens 11) quelqu'un qui ne respecte pas les règles. Les réponses devaient être données selon une échelle de 1 (totalement en accord) à 5 (totalement en désaccord). Un résultat élevé à ces questions indique une identité sociale positive et conventionnelle.

Nombre de partenaires sexuels: les répondantes rapportaient le nombre de partenaires sexuels qu'elles avaient eu au cours de leur vie.

\footnotetext{
vente ou la possession de biens volés, lancer des objets sur des voitures ou des individus, fugue, mentir à propos de son âge, port d'une arme dissimulée, le vol d'objets de moins de $5 \$$, voies de fait graves, prostitution, rapports sexuels, bagarre de gangs, vente de marijuana, tricher aux examens, voies de fait simples, troubler la paix, vente de drogues dures, prise d'un véhicule à moteur sans autorisation, agression sexuelle, vol non armé, être en état d'ébriété sur la voie publique, vol d'objets d'une valeur de 5 à 50 \$, introduction par effraction, faire l'école buissonnière, consommation de drogues et consommation d'alcool.
} 


\section{Variables prédictives}

Les variables relatives au type de délit, à la famille, à l'école et aux pairs décrites ci-dessous ont toutes été mesurées lors du temps 1 de l'étude. À l'exception de quelques-unes, les réponses ont été consignées selon une échelle de Likert à cinq points, soit de «totalement d'accord» à «totalement en désaccord».

Comportements antisociaux antérieurs: les types de délinquants. Tel qu'il a été mentionné précédemment, l'analyse de structure latente a permis de déceler quatre types comportementaux: les conformistes, les délinquantes ayant commis des délits mineurs avec violence, les consommatrices de drogues et les délinquantes qui s'adonnent à une délinquance sérieuse.

Supervision parentale $($ alpha $=0,629)$ - Cette échelle permet d'évaluer dans quelle mesure les parents supervisent les comportements de leurs enfants. Cette échelle contient les trois items suivants: 1) mes parents veulent savoir avec qui je suis lorsque je sors avec d'autres filles 2) dans mes temps libres à l'extérieur de la maison, mes parents savent avec qui et où je suis 3) mes parents veulent savoir où je vais si je ne rentre pas directement à la maison après l'école. Un résultat élevé pour cette échelle indique ainsi une supervision parentale serrée.

Attachement à la famille (alpha $=0,786)$ - Cette échelle a été construite à partir des items suivants : 1) mes parents me demandent souvent ce que je fais à l'école 2) mes parents me donnent suffisamment d'affection 3) une des pires choses qui pourrait m'arriver serait d'apprendre que j'ai déçu mes parents 4) mes parents sont habituellement fiers de moi quand je termine une chose à laquelle j'ai consacré beaucoup d'efforts 5) mes parents me font confiance 6) je suis beaucoup plus proche de mes parents que la plupart des jeunes de mon âge. Un niveau élevé d'attachement à la famille est associé à des résultats élevés pour cette échelle.

Soutien parental au développement de l'identité (alpha $=0,707)$ - Cette échelle comporte les items suivants: 1) mes parents me dénigrent parfois devant d'autres personnes 2) parfois mes parents ne m'écoutent pas ou ne veulent pas connaitre mes opinions 3) mes parents me font parfois sentir que je ne suis pas à la hauteur de leurs attentes 4) mes parents semblent souhaiter que je sois un autre type de personne. Les résultats ont été codifiés de sorte qu'un résultat élevé à cette échelle indique que les parents soutiennent le développement identitaire de l'adolescente.

Engagement des parents envers l'école (alpha $=0,640)$ - Cette échelle constitue une mesure de l'intérêt et du soutien parental à l'égard des 
activités scolaires de l'adolescente. Elle comprend les quatre items suivants : 1) parles-tu souvent des problèmes que tu éprouves à l'école avec tes parents 2) parles-tu souvent avec tes parents de ta relation avec les professeurs 3) parles-tu souvent de tes perspectives de carrière avec tes parents 4) tes parents te demandent-ils souvent ce que tu fais à l'école? Les résultats ont été codifiés de sorte qu'un résultat élevé à cette échelle indique un fort engagement parental à l'égard du fonctionnement scolaire de l'adolescente.

Attachement à l'école (alpha $=0,773)$ - Cette échelle indique jusqu'à quel point la répondante se soucie de l'école et éprouve des sentiments positifs à son endroit. Les deux items suivants forment l'échelle: 1) je ne me sens pas à ma place à l'école 2) j'aimerais bien «décrocher» de l'école. Les résultats ont été codifiés de sorte qu'un résultat élevé à cette échelle indique un fort attachement à l'école.

Engagement à l'école (alpha $=0,753)$ - Cette échelle a été bâtie à partir des items suivants : 1) combien d'heures par semaine consacres-tu à tes devoirs? 2) quelles notes obtiens-tu habituellement à l'école? 3) jusqu'où voudrais-tu te rendre dans tes études? 4) jusqu'où penses-tu te rendre dans tes études? 5) avoir de bonnes notes m'importe peu 6) je travaille fort à l'école 7) le travail que j'accomplis à l'école est très important pour moi 8) les devoirs sont une perte de temps. Les résultats ont été codifiés de sorte qu'un résultat élevé à cette échelle indique un engagement élevé à l'école.

Pressions des pairs (alpha $=0,719)$ - Cette échelle inclut les éléments suivants: 1) je pose parfois des gestes parce que mes amis proches le font 2) je pose parfois des gestes parce que les élèves les plus populaires de mon école le font 3) je pose parfois des gestes parce que mes amis insistent et me harcèlent jusqu'à ce que je cède 4) je n'aime pas être différente ou être à l'écart dans un groupe, c'est pourquoi j'accepte parfois de faire certaines choses 5) je pose parfois des gestes, non pas à cause des pressions de mes amis, mais parce que je crois que je vais les impressionner 6) je pose parfois des gestes pour ne pas perdre le respect de mes amis. La perception d'un niveau élevé de pression de la part des pairs est associée à un résultat élevé sur cette échelle.

Attachement aux pairs (alpha $=0,738)$ - Cette échelle est mesurée par les items suivants: 1) je me sens à l'aise d'appeler mes amis quand j'ai un problème 2) je peux avoir confiance en mes amis, puisque je peux leur dire des choses personnelles tout en sachant qu'ils ne les répéteront pas 3) c'est facile de parler à mes amis 4) mes amis se soucient de 
moi et de ce qui m’arrive. Un résultat élevé à cette échelle indique un fort attachement aux pairs.

Renforcement de la délinquance par les pairs du même sexe $($ alpha $=0,847)$ - Pour construire cette échelle, on a demandé aux répondantes de rapporter jusqu'à quel point leurs amies les encourageraient à : 1) faire des vols à l'étalage 2) voler une voiture pour faire une balade 3) utiliser une fausse carte pour entrer dans un bar 4) participer à une bagarre 5) détruire les biens d'autrui 6) transporter une arme telle qu'un fusil ou un couteau 7) faire l'école buissonnière. L'intervalle des réponses varie de 1 (elles feraient tout pour me décourager) à 5 (elles m'encourageraient fortement). Un résultat élevé témoigne d'un renforcement de la délinquance.

Confiance aux pairs (alpha $=0,693)$ - Les répondantes rapportaient à quelle fréquence elles se confiaient à leurs pairs à propos des enjeux suivants: 1) des problèmes à l'école 2) des relations amoureuses 3) des relations sexuelles 4) de la façon dont les parents les traitent 5) de la compréhension des parents 6) des choses qu'elles ont faites et pour lesquelles elles se sentent coupables. Les réponses suivent un intervalle de 1 (jamais) à 5 (très souvent). Un résultat élévé témoigne d'une forte confiance aux pairs.

\section{Variables contrôles}

Age et race $($ Noirs $=0$, Blancs $=1)-$ ces deux variables sont incluses dans les analyses à titre de variables contrôles.

Pourcentage d'Afro-Américains à l'école - cette variable a été mesurée lors du temps 1 de l'étude. À partir des données du recensement de 1980, la composition raciale des écoles fréquentées par les répondantes a été évaluée.

Niveau de scolarité - Cette variable a été mesurée lors du temps 2 de l'étude et son intervalle varie de 1 (moins qu'un niveau d'études secondaires) à 5 (4 ans et plus d'études collégiales).

Statut occupationnel - Cette variable a également été mesurée lors du temps 2 et constitue un classement général du prestige de l'emploi occupé. Les occupations rapportées par les répondants vont de 1 (personnel de direction, administrateurs, gestionnaires) à 7 (employés du secteur des services et ouvriers).

Statut d'emploi - Une seule question du temps 2 forme cette variable: Occupez-vous un emploi présentement? Les réponses sont 0 (non, je n'occupe pas d'emploi) et 1 (oui, j'occupe un emploi).

Revenu du ménage - Mesurée lors du temps 2 de l'étude, cette variable s'échelonne sur 11 catégories qui varient de 1 (moins de 7000 \$ par année) à 11 (50 $000 \$$ et plus par année). 


\section{Analyse et résultats}

Modélisation des facteurs associés à la délinquance des adolescentes

En guise de première étape, les corrélations liés à la famille, à l'école et aux pairs ont été estimées en regard des échelles pondérées de la délinquance juvénile ${ }^{5}$. Les coefficients de la régression multiple du tableau 1 révèlent que l'attachement à l'école réduit les risques d'implication dans la délinquance, alors que le renforcement de la délinquance par les pairs augmente ces mêmes risques. Aucune autre variable du modèle ne constitue un prédicteur significatif. Puisque ce modèle ne s'applique peut-être pas à tous les types de délinquantes, les distinctions entre les adolescentes conformistes et les délinquantes des trois autres types (adolescentes ayant commis des délits mineurs, consommatrices de drogues et délinquantes ayant commis des délits graves) ont été examinées selon des dimensions personnelles et sociales (tableau 2). Étant donné

TABLEAU 1

Coefficients non standardisés pour la régression multiple sur la délinquance juvénile en fonction des variables de la famille, de l'école, des pairs et des variables contrôles

\begin{tabular}{|lc|}
\hline Prédicteurs & Échelle de délinquance juvénile pondérée \\
\hline Constante & 10,733 \\
Race (Blancs) & $-4,712$ \\
Pourcentage d'Afro-Américains à l'école & $-0,057$ \\
Âge & 0,298 \\
Attachement à la famille & $-0,709$ \\
Supervision parentale & 0,452 \\
Soutien au développement de l'identité & $-1,974$ \\
Engagement à l'école & 0,798 \\
Attachement à l'école & $-3,872^{* *}$ \\
Attachement aux pairs & $-0,277$ \\
Pressions des pairs & 1,868 \\
Renforcement de la délinquance par les pairs & $7,582^{* *}$ \\
\multicolumn{1}{c}{$\left(R^{2}\right)$} & 0,345 \\
\hline
\end{tabular}

Notes: $\mathrm{n}=367$

${ }^{*} p<0,05,{ }^{* *} p<0,01$

5. L'annexe 2 présente la matrice de corrélation pour les variables de la famille, de l'école et des pairs incluses dans les analyses. 
que la variable dépendante est catégorielle et hautement asymétrique, un logit multinomial a été utilisé pour estimer les effets de ces variables pour les quatre catégories de délinquantes. En effet, la régression logistique multinomiale permet une analyse d'une variable dépendante nominale à plusieurs catégories. D'après Long (1997), la régression logistique multinominale peut se comprendre comme une estimation simultanée des logits binaires pour l'ensemble des comparaisons possibles entre les groupes. Les coefficients estiment ainsi l'effet des prédicteurs sur les probabilités qu'une adolescente soit classifiée dans un type particulier de délinquance.

Le tableau 2 présente une estimation des effets des variables retenues pour les quatre catégories de délinquantes. La première équation met en opposition la délinquance grave et le conformisme, la deuxième équation met en opposition l'usage de drogues et le conformisme, et enfin, la troisième équation met en opposition la délinquance mineure avec violence et le conformisme. Les résultats peuvent être adéquatement résumés par les différents effets résultant des contrastes entre les quatre catégories de délinquantes. La deuxième colonne du tableau 2 indique les résultats au test du khi-carré pour les effets globaux de chaque variable. Long et McGinnis (1981) soutiennent qu'à l'intérieur d'une régression logistique multinomiale, les tests individuels et spécifiques de signification doivent être analysés en même temps que l'ensemble des effets du modèle (et de leur signification globale) pour une variable indépendante donnée. Cela permet d'évaluer le potentiel de chaque variable indépendante à différencier les catégories de délinquantes. Les résultats indiquent que le soutien parental au développement de l'identité, l'attachement aux pairs et le renforcement de la délinquance par les pairs sont significativement associés à la délinquance et, ce qui est encore plus important, qu'ils distinguent les types de délinquantes.

Par opposition aux résultats de l'échelle de délinquance pondérée présentés au tableau 1, les modèles présentés au tableau 2 indiquent que le soutien parental au développement de l'identité réduit significativement l'implication des adolescentes dans la délinquance pour l'ensemble des délinquantes. Les effets de cette relation sont particulièrement importants chez les consommatrices de drogues et un peu plus limités chez les adolescentes ayant commis des délits mineurs avec violence. Comme le mentionnent Stein et Newcomb (1999), un tel résultat s'avère particulièrement important lorsqu'on sait que la formation d'une identité saine au début de l'adolescence favorise le développement de 
TABLEAU 2

Coefficients logit et rapports de cotes issus de la régression logistique multinomiale sur la délinquance à l'adolescence en fonction des variables relatives à la famille, à l'école, aux pairs et aux variables contrôles

\begin{tabular}{|c|c|c|c|c|c|c|c|}
\hline \multirow[b]{3}{*}{ Prédicteurs } & & \multicolumn{2}{|c|}{ Équation 1} & \multicolumn{2}{|c|}{ Équation 2} & \multicolumn{2}{|c|}{ Équation 3} \\
\hline & & \multicolumn{2}{|c|}{$\begin{array}{c}\text { Délinquance } \\
\text { grave } \\
\text { vs conformisme }\end{array}$} & \multicolumn{2}{|c|}{$\begin{array}{l}\text { Consommation } \\
\text { de drogues } \\
\text { vs conformisme }\end{array}$} & \multicolumn{2}{|c|}{$\begin{array}{c}\text { Déliquance } \\
\text { mineure avec } \\
\text { violence } \\
\text { vs conformisme }\end{array}$} \\
\hline & $\begin{array}{c}X^{2} \\
\text { (global) }\end{array}$ & B & $\operatorname{Exp}(B)$ & B & $\operatorname{Exp}(B)$ & B & $\operatorname{Exp}(B)$ \\
\hline Ordonnée à L'origine & & 0,707 & & $-4,629$ & & 2,122 & \\
\hline$\%$ d'Afro-Américains & 2,15 & $-0,007$ & 0,993 & $-0,004$ & 0,996 &,- 007 & 0,993 \\
\hline Race (Blancs) & 0,45 & $-0,215$ & 1,240 & $-0,242$ & 1,274 &,- 144 & 1,154 \\
\hline Âge & 16,30 ** & 0,164 & 1,179 & $0,298^{* *}$ & 1,347 &,- 032 & 0,969 \\
\hline Attachement familial & 2,22 & $-0,112$ & 0,894 & $-0,421$ & 0,657 &,- 228 & 0,796 \\
\hline Supervision parentale & 0,88 & $-0,251$ & 0,778 & 0,003 & 1,003 &,- 077 & 0,926 \\
\hline Soutien parental & 13,39 ** & $-0,671^{*}$ & 0,511 & $-0,707^{* *}$ & 0,493 &,$- 506^{* *}$ & 0,603 \\
\hline Engagement à l'école & 5,08 & $-0,696$ & 0,499 & $-0,207$ & 0,813 & 117 & 1,124 \\
\hline Attachement à l'école & 4,21 & $-0,540^{*}$ & 0,583 & $-0,263$ & 0,768 &,- 200 & 0,819 \\
\hline Attachement aux pairs & 12,22 ** & 0,367 & 1,443 & $0,870^{*}$ & 2,387 & ,494 & 1,638 \\
\hline Pressions des pairs & 1,12 & 0,299 & 1,348 & 0,151 & 1,163 & 157 & 1,170 \\
\hline Renforcement des pairs & $29,01^{* *}$ & $1,156^{* *}$ & 3,178 & 0,869 ** & 2,385 & ,302 & 1,353 \\
\hline Khi-carré du modèle & 147,589 & & & & & & \\
\hline
\end{tabular}

Notes: $\mathrm{n}=367$

${ }^{*} p<0,05, * * p<0,01$

l'intimité à l'aube de l'âge adulte, ce qui encourage par conséquent le développement de relations interpersonnelles et laisse entrevoir une plus grande satisfaction de vivre.

Les modèles 1 et 2, par ailleurs, démontrent que les adolescentes sont plus susceptibles de s'engager dans une délinquance grave et dans la consommation de drogues lorsqu'elles ont la perception que leurs pairs renforcent leurs comportements délinquants. Le modèle 3, quant à lui, montre que ce renforcement par les pairs n'a pas d'effet significatif sur la commission de délits mineurs avec violence. Le modèle 1 fait ressortir que l'attachement à l'école tend à réduire le risque que l'adolescente ne s'engage dans le type le plus sérieux de délinquance (par rapport au type conformiste), mais les modèles 2 et 3 révèlent que cette variable n'a aucun effet significatif sur les deux autres types de délinquance. Finalement, le modèle 2 démontre que le risque de consommer de la drogue 
augmente (par rapport au type conformiste) chez les adolescentes lorsqu'elles sont fortement attachées à leurs pairs. Toutefois, comme l'indiquent les résultats des modèles 1 et 3 , ces relations attentionnées avec les pairs ne semblent pas avoir d'effet significatif sur la délinquance grave ou la commission de délits mineurs avec violence.

Les résultats mettent en évidence l'influence considérable des variables liées aux parents et aux pairs sur la consommation de drogues et sur la délinquance grave. Puisque l'analyse de structure latente révélait l'implication marquée des délinquantes sérieuses dans les activités reliées à la drogue, des analyses supplémentaires ont été effectuées afin d'isoler l'effet de la consommation de drogues sur les comportements délinquants. L'influence des variables retenues a donc été vérifiée sur une mesure dichotomique de la consommation de drogues. Puisque les délinquantes sérieuses sont très impliquées dans des activités de consommation et de vente de drogues, l'utilisation de cette variable dichotomique permet de passer de deux catégories (délinquantes sérieuses et consommatrices de drogues) à une seule et même catégorie. Les résultats du

TA B LEA U 3

Coefficients logit et rapports de cotes pour la régression portant sur la consommation de drogues à l'adolescence en fonction des variables de la famille, de l'école, des pairs et des variables contrôles

\begin{tabular}{|llc|}
\hline & \multicolumn{2}{c|}{ Consommation de drogues } \\
\cline { 2 - 3 } Prédicteurs & Coefficients & Rapports de cotes \\
\hline Constante & $-6,515^{* *}$ & \\
Race (blanche) & 0,095 & 1,100 \\
\% d'Afro-Américains à l'école & 0,006 & 1,006 \\
Âge & $0,367^{* *}$ & 1,444 \\
Attachement à la famille & $-0,026$ & 0,974 \\
Supervision parentale & $-0,264$ & 0,768 \\
Soutien parental à l'identité & $-0,516^{* *}$ & 0,597 \\
Engagement à l'école & $-0,339$ & 0,713 \\
Attachement à l'école & $-0,279$ & 0,757 \\
Attachement aux pairs & $0,687^{* *}$ & 1,987 \\
Pressions des pairs & 0,002 & 1,002 \\
Renforcement de la délinquance par les pairs & $0,725^{* *}$ & 2,064 \\
Khi-carré du modèle & 105,016 & \\
\hline
\end{tabular}

Notes: $\mathrm{n}=367$

${ }^{*} p<0,05,{ }^{* *} p<0,01$ 
tableau 3 sont tout à fait cohérents avec ceux du tableau 2, qui portaient sur les quatre catégories de délinquantes. En effet, il apparaît que le soutien parental au développement de l'identité protège, en définitive, les adolescentes contre la consommation de drogues, tout comme pour les délits violents et les délits contre la propriété qui s'allient à la consommation de drogues; bref, elle diminue les probabilités de s'engager dans la délinquance. Les résultats indiquent également qu'un attachement étroit aux pairs et que le renforcement de la délinquance par ces derniers favorise les activités reliées à la drogue. De tels résultats démontrent la nécessité de s'attarder davantage à la problématique de la consommation de drogues des adolescentes et d'étudier plus spécifiquement ses conséquences au moment de la transition vers l'âge adulte.

\section{Modélisation des conséquences de la délinquance juvénile}

À l'étape suivante, les conséquences de l'adoption de divers comportements délinquants au cours de l'adolescence sur l'adaptation sociale à l'âge adulte ont été examinées. Une variété d'indicateurs mesurés à l'âge adulte - comportements criminels, identité sociale, comportements sexuels, consommation d'alcool/drogues - a été évaluée pour trois catégories de délinquantes (variables dichotomisées), en utilisant les adolescentes conformistes comme catégorie de comparaison. Les variables sociodémographiques, celles de la famille, de l'école et des pairs ont été utilisées à titre de variables contrôles ${ }^{6}$. Les variables contrôles sont les suivantes : âge, race, niveau de scolarité, prestige occupationnel, revenu du ménage, statut d'emploi, soutien parental au développement de l'identité à l'adolescence, supervision parentale, attachement à la famille, engagement des parents envers l'école, engagement à l'école, pressions des pairs, attachement aux pairs et confiance aux pairs.

Le modèle 1 du tableau 4 fait état de l'influence des comportements délinquants à l'adolescence sur les comportements criminels à l'âge

6. Nous avons mesuré des modèles supplémentaires qui incluaient l'état matrimonial, la satisfaction dans le mariage et le statut parental. Ces variables n'étaient pas significativement corrélées aux types de délinquantes. Ainsi, leur inclusion dans les modèles présentés n'ont eu aucun effet sur les autres variables du modèle. Également, compte tenu du grand nombre de valeurs manquantes pour ces variables, elles n'ont pas été incluses dans les modèles présentés ci-dessous. 
adulte $^{7}$. Les résultats indiquent que parmi les quatre types de délinquance dégagés par l'analyse de structure latente, seule la délinquance grave durant l'adolescence est associée à un comportement criminel à l'âge adulte. Un autre modèle a été conçu dans le but d'examiner les effets de la délinquance juvénile en utilisant les 27 items de l'échelle des comportements délinquants (modèle non présenté). L'effet de cette échelle de délinquance pondérée sur les comportements criminels à l'âge adulte n'est pas significatif. C'est donc dire que seule la délinquance grave est significativement associée aux comportements criminels à l'âge adulte. De tels résultats démontrent la nécessité de poursuivre la recherche sur l'influence particulière et distincte, sur le cours de leur vie, des différents types de délinquance à l'adolescence. Enfin, la criminalité adulte s'explique aussi par une supervision parentale relâchée durant l'adolescence et par le fait d'être sans emploi à l'âge adulte.

Le modèle 2 du tableau 4 examine les conséquences de la délinquance à l'adolescence sur le développement de l'identité sociale à l'âge adulte. Les résultats indiquent que tous les types de délinquance réduisent les chances de développement d'une identité sociale conventionnelle à l'âge adulte. Cet effet est un plus marqué dans le cas de la délinquance grave, bien que les trois types de délinquance aient un effet relativement similaire sur l'identité sociale à l'âge adulte. D'autre part, seul le revenu du ménage s'ajoute à la délinquance juvénile comme prédicteur de l'identité sociale à l'âge adulte. Étant donné que l'implication dans des activités liées à la drogue caractérise tant les consommatrices de drogues que les délinquantes sérieuses, les répercussions de la consommation de drogues à l'adolescence ont été analysées de plus près. Un modèle (non présenté ici) a été estimé à partir d'une mesure dichotomique de la consommation de drogues. Les résultats indiquent que toute activité liée à la consommation de drogues à l'adolescence (ayant

7. Nos données contiennent une mesure rétrospective et autorévélée de la délinquance manifestée avant l'âge de 21 ans, mesure que nous avons utilisée pour valider l'analyse de structure latente. Nous avons constaté que moins de 20 répondantes ont été mal classées dans la catégorie des adolescentes conformistes, et ce, sur la base de 16 questions portant sur la délinquance individuelle. Les délits qui ont été le plus souvent mal classés sont la consommation de drogues et les délits mineurs avec violence (frapper quelqu'un). Dans nos analyses multivariées, nous avons décidé de laisser ces répondantes dans le groupe des adolescentes conformistes. Nous avons également réestimé les modèles multivariés en excluant ces répondantes (ces modèles ne sont pas présentés dans cet article, mais peuvent être obtenus des auteurs). Les résultats indiquent qu'il n'y a pas de différence statistiquement significative ou substantielle en comparaison avec les modèles présentés précédemment. 
TABLEAU 4

Estimations OLS non standardisées pour l'analyse de régression des comportements criminels à l'âge adulte et de l'identité sociale en fonction de la délinquance juvénile et des variables contrôles

\begin{tabular}{|lcc|}
\hline & Modèle 1 & Modèle 2 \\
\cline { 2 - 3 } Prédicteurs & $\begin{array}{c}\text { Échelle de } \\
\text { délinquance pondérée }\end{array}$ & $\begin{array}{c}\text { Identité } \\
\text { sociale }\end{array}$ \\
\hline Constante & $11,988^{* *}$ & $2,475^{* *}$ \\
Consommatrices de drogues & 0,207 & $-0,250^{* *}$ \\
Délinquantes s'adonnant à la violence mineure & $-0,001$ & $-0,255^{* *}$ \\
Délinquantes s'adonnant à la délinquance grave & $0,978^{*}$ & $-0,288^{* *}$ \\
Statut d'emploi (oui) & $-0,835^{* *}$ & 0,067 \\
Âge & $-0,128$ & 0,021 \\
Race (Blancs) & $-0,568^{*}$ & $-0,052$ \\
Niveau de scolarité & 0,012 & $-0,017$ \\
Catégorie professionnelle & 0,006 & $-0,011$ \\
Revenu par ménage & 0,029 & $0,017^{*}$ \\
Soutien parental à l'identité & 0,034 & $-0,005$ \\
Supervision parentale & $-0,611^{* *}$ & 0,031 \\
Attachement à la famille & $-0,056$ & 0,041 \\
Engagement des parents envers l'école & 0,213 & $-0,016$ \\
Engagement à l'école & 0,073 & 0,072 \\
Pressions des pairs & $-0,271$ & 0,044 \\
Confiance aux pairs & 0,096 & 0,020 \\
Attachement aux pairs & 0,287 & 0,019 \\
$R^{2}$ & 0,344 & 0,420 \\
\hline
\end{tabular}

Notes: $\mathrm{n}=367$

${ }^{*} p<0,05,{ }^{* *} p<0,01$

ou non donné lieu à d'autres comportements criminels) est négativement associée à une identité sociale conventionnelle au début de l'âge adulte. Conséquemment, il apparait clairement que chez les adolescentes, la consommation et/ou la vente de drogues influent négativement sur nombre de situations à l'âge adulte. Considérant les conséquences caractéristiques d'une consommation de drogues à l'adolescence sur la transition à la vie adulte, les analyses subséquentes ont accordé une importance particulière aux consommatrices de drogues.

Ensuite, les conséquences des comportements délinquants à l'adolescence ont été analysées en fonction de deux autres enjeux sociaux et 
comportementaux à l'âge adulte: le comportement sexuel et la consommation de drogues et d'alcool. En plus d'un effet significatif de la race, le modèle 1 du tableau 5 indique qu'une implication dans n'importe quelle des catégories de délinquance à l'adolescence augmente le nombre de partenaires sexuels à l'âge adulte. Plus encore, les coefficients de régression standardisés (non présentés ici) démontrent que cette relation entre la délinquance juvénile et les comportements sexuels futurs est plus marquée lorsqu'il y a consommation de drogues à l'adolescence. Cette constatation s'accorde avec les résultats des recherches de Parker et al. (1994) et de Smith et Brown (1998), lesquels montrent qu'il existe un

\section{TA B LEA U 5}

Estimations OLS non standardisées pour l'analyse de régression des comportements sexuels et de la consommation abusive d'alcool et de drogues à l'âge adulte en fonction des types de délinquance à l'adolescence et des variables contrôles

\begin{tabular}{|lcc|}
\hline Prédicteurs & Modèle 1 & Modèle 2 \\
\cline { 2 - 3 } & $\begin{array}{c}\text { Comportements } \\
\text { sexuels }\end{array}$ & $\begin{array}{c}\text { Consommation } \\
\text { abusive d'alcool } \\
\text { et de drogues }\end{array}$ \\
\hline Constante & $8,578^{*}$ & 0,527 \\
Consommatrices de drogues & $1,479^{* *}$ & $0,207^{* *}$ \\
Délinquantes s'adonnant à la violence mineure & $0,798^{*}$ & $0,135^{* *}$ \\
Délinquantes s'adonnant à la délinquance grave & $1,573^{*}$ & $0,233^{* *}$ \\
Statut d'emploi (oui) & $-0,324$ & $-0,062$ \\
Âge & $-0,134$ & $-0,018$ \\
Race (Blancs) & $-0,658^{*}$ & $-0,005$ \\
Niveau de scolarité & $-0,093$ & $0,056^{*}$ \\
Prestige occupationnel & 0,067 & 0,002 \\
Revenu du ménage & $-0,091$ & $-0,006$ \\
Soutien parental à l'identité & 0,200 & $0,057^{*}$ \\
Supervision parentale & $-0,075$ & $-0,008$ \\
Attachement à la famille & $-0,502$ & $-0,125^{* *}$ \\
Engagement des parents envers l'école & $-0,445$ & 0,002 \\
Engagement à l'école & 0,416 & 0,082 \\
Pressions des pairs & 0,283 & 0,010 \\
Confiance aux pairs & 0,129 & 0,001 \\
Attachement aux pairs & 0,215 & $-0,019$ \\
$R^{2}$ & 0,334 & 0,357 \\
\hline Note $N$ (367 & & \\
\hline
\end{tabular}

Note: $N=367$

${ }^{*} p<0,05,{ }^{* *} p<0,01$ 
lien entre une importante consommation d'alcool et de drogues et les comportements sexuels à risque. Ce lien entre la consommation de drogues et les comportements sexuels avec de multiples partenaires peut avoir d'importantes répercussions sur la santé gynécologique et la fertilité de la femme.

Le modèle 2 du tableau 5 indique que le niveau de scolarité, le soutien parental au développement de l'identité, ainsi que l'attachement à la famille à l'adolescence sont tous des prédicteurs significatifs de l'abus d'alcool et de drogues à l'âge adulte. Si l'effet positif du niveau de scolarité sur la consommation d'alcool et de drogues s'accorde bien avec les résultats des recherches sur l'usage modéré d'alcool à l'âge adulte, ce lien concorde moins avec les études effectuées dans les domaines de la toxicomanie et de l'alcoolisme (DeMaris et Kaukinen, 2005; Kaukinen, 2002). L'effet positif du soutien parental au développement d'une identité reflète peut-être le lien existant entre le comportement des parents et leur rôle de modèle en matière de consommation d'alcool. Certaines recherches ont par exemple démontré que les pratiques, les normes et les attitudes des parents concernant la consommation d'alcool et de drogues sont fortement associées à la consommation d'alcool des adolescents et à leurs répercussions connexes (Hawkins et al., 1997).

Plus important encore, les résultats indiquent que si tous les types de délinquance juvénile augmentent le risque d'une consommation abusive d'alcool et de drogues à l'âge adulte, la comparaison des coefficients standardisés (non présentés ici) démontre encore une fois que ce sont les consommatrices de drogues à l'adolescence qui sont le plus à risque d'abuser de substances psychotropes à l'âge adulte. Comparativement aux adolescentes conformistes et aux deux autres types de délinquantes, les adolescentes consommatrices de drogues risquent davantage de connaître des problèmes d'alcool et de drogues à l'âge adulte. Ce résultat est conforme aux travaux de Chassin et al. (2002), qui rapportent que l'abus de substances psychotropes à l'adolescence augmentait le risque d'abus ou de dépendance à l'alcool ou aux drogues dans le futur. Considérant cette stabilité, il apparaît clairement que la consommation abusive de drogues à l'adolescence ne doit pas être ignorée. Mis tous ensemble, ces résultats renferment d'importantes considérations pour les politiques en matière de santé publique. Étant donné le chevauchement de la consommation de drogues et de l'initiation précoce à la sexualité, les initiatives en matière de santé publique devraient tenir compte des conséquences de ces comportements sur la santé globale et 
le bien-être des femmes. La consommation de drogues à l'adolescence constitue certainement un point tournant pour bon nombre de comportements ultérieurs.

\section{Sommaire et discussion}

Au cours des dernières années, les délinquants persistants ont été le centre d'intérêt de la recherche criminologique développementale, et ce, au détriment d'autres types qualitatifs de délinquants. Nos analyses indiquent qu'il existe d'importantes différences entre les formes de la délinquance des adolescentes, notamment entre les types distincts de délinquantes. Ces types incluent les adolescentes qui ne s'adonnent qu'à des délits violents de faible gravité, les consommatrices de drogues et les délinquantes qui posent des gestes plus graves. Qui plus est, nous avons constaté que ces divers types de délinquance témoignaient d'un certain nombre de caractéristiques étiologiques tantôt communes, tantôt uniques. Le soutien parental au développement d'une identité semble agir comme un important facteur de protection pour chacun des types de délinquance chez les adolescentes, alors que le renforcement de la délinquance par les pairs est associé à la consommation de drogues et à la délinquance grave. L'attachement à l'école apparaît être un facteur de protection uniquement pour la délinquance grave. Dans la même veine que les recherches qui attestent que le décrochage scolaire favorise la persistance des activités antisociales tant chez les garçons que chez les filles, nos résultats concernant l'engagement à l'école encouragent fortement le déploiement d'efforts afin d'encourager les adolescentes à poursuivre leurs études. Nos données indiquent également que les adolescentes dont les activités sont principalement liées aux drogues présentent des caractéristiques distinctes des autres délinquantes. En effet, il semble que pour ce type de délinquantes, la consommation de drogues soit associée à un fort attachement aux pairs, ce qui n'est pas le cas pour les autres types de délinquantes.

Par ailleurs, notre recherche a mis en évidence l'importance de déceler les facteurs de risque liés à la délinquance des adolescentes, ainsi que les facteurs de protection susceptibles de préserver ces dernières de la délinquance et de ses conséquences à long terme. Le conformisme des adolescentes est clairement associé à une supervision parentale étroite, à l'attachement aux parents, ainsi qu'au soutien parental au développe- 
ment de l'identité. Ces adolescentes, qui ne rapportent pas de participation aux activités délinquantes, sont également bien intégrées aux activités scolaires et bien décidées à poursuivre leurs études. Ces facteurs de protection semblent les empêcher de s'engager dans la délinquance. Plus important encore, le conformisme des adolescentes favorise une bonne adaptation à la vie adulte, qui se traduit par une faible participation à des activités délinquantes et par une identité sociale consolidée. Il est également important de souligner que l'absence de consommation de drogues et d'alcool chez les adolescentes se traduit aussi par une faible consommation de telles substances à l'âge adulte. L'absence de consommation de psychotropes réduit également l'adoption de comportements à risque, tel que le fait d'avoir eu de nombreux partenaires sexuels. En somme, il apparaît clairement que le fait d'assurer une supervision attentionnée aux adolescentes, de promouvoir chez elles une identité sociale positive et de les encourager à s'engager activement à l'école sont d'importants mécanismes qui réduisent la consommation de drogues et d'autres activités délinquantes, tout en favorisant une transition plus harmonieuse de l'adolescence à l'âge adulte.

Nos résultats nous apparaissent suffisamment étoffés pour affirmer que la consommation de drogues a des conséquences certaines, à l'âge adulte, sur l'usage de substances psychotropes et les comportements sexuels. Considérant que la consommation de drogues a des répercussions majeures sur le développement et le maintien de relations interpersonnelles (Newcomb et Jack, 1995), on ne saurait trop insister sur le rôle des parents, des professeurs et des intervenants dans le dépistage des problèmes précoces de consommation de drogues et d'alcool des adolescentes. Gorman (1996) affirme que des programmes devraient être conçus pour cibler les jeunes qui manifestent des symptômes précoces de surconsommation de drogues. Les approches ainsi proposées pourraient alors se concentrer sur la détermination des comportements délinquants précoces, sur les activités antisociales et sur les difficultés scolaires. Nos résultats indiquent également que l'implication dans une délinquance grave durant l'adolescence est associée à un faible engagement à l'école, à une surveillance parentale relâchée et à une gamme d'influences criminogènes provenant des pairs, autant de conditions qui augmentent le risque de comportements criminels à l'âge adulte. Les facteurs de risque déterminés dans notre étude correspondent à ce que Loeber and Farrington (1998) ont dégagé comme principales composantes d'un programme de prévention s'adressant à la délinquance dite 
chronique et sérieuse. Ces composantes sont : 1) renforcer les habiletés des parents en matière de supervision et de contrôle des enfants, tout en consolidant leurs valeurs morales 2) soutenir les institutions sociales centrales, telles que les écoles et les organismes communautaires 3) promouvoir des programmes de prévention de la délinquance qui dépistent spécifiquement les jeunes les plus à risque et qui s'adressent à eux 4) intervenir rapidement lorsque se manifestent des comportements délinquants en recourant à un système de sanctions progressives, à des services continus et à des sanctions intermédiaires et communautaires.

\section{Références}

Adler, F. (1975). Sisters in crime. New York: McGraw-Hill.

Ageton, S. (1983). The dynamics of female delinquency, 1976-1980. Criminology, 21, 555-584.

Agnew, R. (1984). Autonomy and delinquency. Sociological Perspectives, 27 (2), 219-240.

Barker, G.H., \& Adams, W.T. (1962). Comparison of the delinquencies of boys and girls. Journal of Criminal Law, Criminology, and Police Science, 53, 470-475.

Berger, R. (1989). Female delinquency in the emancipation era: a review of the literature. Sex Roles, 21, 375-399.

Boyer, D., \& Fine, D. (1992). Sexual abuse as a factor in adolescent pregnancy. Family Planning Perspectives, 24, 4-11.

Blumstein, A., Cohen, J., \& Farrington, D. (1988). Criminal careers research : its value for criminology. Criminology, 26, 1-35.

Brownfield, D., \& Sorenson, A. (1987). A latent structure of delinquency. Journal of Quantitative Criminology, 3 (2), 103-124.

Bureau of the Census (1980). Alphabetical index of industries and occupations (1st edition), 1980 census of population. U.S. Department of Commerce, Washington, DC, Government Printing Office.

Campbell, A. (1981). Girl delinquents. New York: St. Martin's Press.

Cernkovich, S., \& Giordano, P. (1979). A comparative analysis of male and female delinquency. The Sociological Quarterly, 20, 131-145

Cernkovich, S., \& Giordano, P. (1992). School bonding, race, and delinquency. Criminology, 30, 261-291.

Cernkovich, S., \& Giordano, P. (2001). Stability and change in antisocial behavior: the transition from adolescence to early adulthood. Criminology, 39, 371-410.

Chassin, L., Pitts, S., \& Prost, J. (2002). Binge drinking trajectories from adolescence to emerging adulthood in a high-risk sample: predictors and substance abuse outcomes. Journal of Consulting \& Clinical Psychology, 70 (1), 67-78. 
Chung, I., Hill, K., Hawkins, J.D., Gilchrist, L., \& Nagin, D. (2002). Childhood predictors of offense trajectories. Journal of Research in Crime and Delinquency, 39, 60-90.

Clinard, M., \& Quinney, R. (1973). Criminal behavior systems: a typology, 2nd edition. New York: Holt, Rinehart and Winston.

Côté, S., Zoccolillo, M., Tremblay, R., Nagin, D., \& Vitaro, F. (2001). Predicting girls' conduct disorder in adolescence from childhood trajectories of disruptive behaviors. Journal of the American Academy of Child and Adolescent Psychiatry, 40, 678-684.

DeMaris, A., \& Kaukinen, C. (2005). Violent victimization and women's mental and physical health. Journal of Research in Crime and Delinquency, à paraître.

Dunforth, F., \& Elliott, D. (1984). Identifying career offenders using selfreported data. Journal of Research in Crime and Delinquency, 27, 57-86.

D'Unger, A., Land, K., \& McCall, P. (2002). Sex differences in age patterns of delinquent/criminal careers : results from Poisson latent class analyses of the Philadelphia cohort study. Journal of Quantitative Criminology, 18, 349-375.

D’Unger, A., Land, K., McCall, P., \& Nagin, D. (1998). How many latent classes of delinquent/criminal careers? Results from mixed Poisson regression analysis. American Journal of Sociology, 103, 1593-1630.

Elliott, D., \& Ageton, S. (1980). Reconciling race and class differences in selfreported and official estimates of delinquency. American Sociological Review, 45, 95-110.

Elliott, D., David, H., \& Ageton, S. (1985). Explaining delinquency and drug use. Beverly Hills, CA : Sage.

Farrington, D., \& Hawkins, J.D. (1991). Predicting participation, early onset and later persistence in officially recorded offending. Criminal Behaviour and Mental Health, 1, 1-33.

Fergusson, D., Horwood, J., \& Nagin, D. (2000). Offending trajectories in a New Zealand birth cohort. Criminology, 38, 525-551.

Fergusson, D., Lynskey. M., \& Horwood, J. (1996). Factors associated with continuity and changes in disruptive behavior. Journal of Abnormal Child Psychology, 24, 533-553.

Gibbons, D. (1985). The assumption of the efficacy of middle-range explanations : typologies. In R. Meier (ed.), Theoretical Methods in Criminology. Beverly Hills, CA : Sage.

Gorman, D.M. (1996). Etiological theories and the primary prevention of drug use. Journal of Drug Issues, 26 (2), 505-521.

Gottfredson, M., \& Hirschi, T. (1986). The true value of lambda would appear to be zero: an essay on career criminals, criminal careers, selective incapacitation, cohort studies, and related topics. Criminology, 24, 213-234.

Giordano, P., \& Cernkovich, S. (1979). On complicating the relationship between liberation and delinquency. Social Problems, 26, 467-481.

Hawkins, J.D., Graham, J., Maguin, E., Abbott, R., Hill. K., \& Catalano, R. (1997). Exploring the effects of age of alcohol use initiation and psychosocial risk factors on subsequent alcohol misuse. Journal of Studies on Alcobol, 58, 280290. 
Heinen, T. (1996). Latent class analysis and discrete latent trait models: similarities and differences. Thousand Oaks, CA: Sage Publications.

Kaukinen, C. (2002). Adolescent victimization and problem drinking. Violence and Victim, 17, 669-689.

Lazarsfeld, P., \& Henry, N. (1965). Latent structure analysis. Boston: HoughtonMifflin.

Le Blanc, M. (1997). A generic control theory of the criminal phenomenon: the structural and dynamic statements of an integrative multilayered control theory. In T. Thornberry (ed.), Developmental theories of crime and delinquency (215-85). New Brunswick, NJ: Transaction Publications.

Lanctôt, N., \& Le Blanc, M. (2002). Les trajectoires marginales chez les adolescentes judiciarisées: continuité et chargement. Revue Internationale de Criminologie et de Police Technique et Scientifique, 53, 46-68.

Lanctôt, N., \& Le Blanc, M. (2002). Explaining deviance by adolescent females. In M. Tonry (ed.), Crime and Justice: A Review of Research, 29 (113-202). Chicago: University of Chicago Press.

Loeber, R. (1996). Developmental continuity, change, and pathways in male juvenile problem behaviors and delinquency. In J.D. Hawkins (ed.), Delinquency and crime: current theories (1-27). New York: Cambridge University Press.

Loeber, R., \& Le Blanc, M. (1990). Toward a developmental criminology. In M. Tonry et N. Morris (eds), Crime and justice: an annual review of research, vol. 12. Chicago, IL: University of Chicago Press.

Loeber, R., \& Farrington, D. (1998). Never too early, never too late: risk factors and successful interventions for serious and violent juvenile offenders. Studies on Crime and Crime Prevention, 7 (1), 7-30.

Loeber, R., Stouthamer-Loeber, M., Van Kammen, W., \& Farrington, D. (1991). Initiation, escalation and desistance in juvenile offending and their correlates. Journal of Criminal Law and Criminology, 82, 36-82.

Lombroso, C., \& Ferrero, W. (1916). The female offender. New York : Philosophical Society.

Long, S. (1997). Regression models for categorical and limited dependent variables. Thousand Oaks, CA: Sage Publications.

Long, S., \& McGinnis, R. (1981). Organization Context and Scientific Productivity. American Sociological Review, 46, 422-442.

McCord, J. (1993). Gender issues. In C. Culliver (ed.), Female criminality: the state of the art (105-118). New York: Garland.

McCutcheon, A. (1987). Latent class analysis. Newbury Park : Sage.

McLachlan, G., \& Basford, K. (1988). Misture models: inference and applications to clustering. New York: M. Dekker.

Moffitt, T. (1993). Adolescent limited and life course-persistent antisocial behavior: a developmental taxonomy. Psychological Review, 100, 674-701.

Moffitt, T. (1997). Neuropsychology, antisocial behavior, and neighborhood context. In J. McCord (ed.), Violence and childhood in the inner city. Cambridge: Cambridge University Press. 
Moffitt, T., Caspi, A., Dickson, N., Silva, P., \& Stanton, W. (1996). Childhoodonset versus adolescent-onset antisocial conduct in males: natural history from age 3 to 18. Development and Psychopathology, 8, 399-424.

Nagin, D., \& Land, K. (1993). Age, criminal careers, and population heterogeneity: specification and estimation of a nonparametric, mixed Poisson model. Criminology, 31, 327-362.

Nagin, D., \& Paternoster, R. (1991). On the relationship of past and future participation in delinquency. Criminology, 29, 163-189.

Nagin, D., \& Paternoster, R. (2000). Population heterogeneity and state dependence: state of the evidence and directions for future research. Journal of Quantitative Criminology, 16, 117-144.

Newcomb, M., \& Jack, L. (1995). Drug use, agency, and communality : causes and consequences among adults. Psychology of Addictive Behaviors, 9 (1), 67-82.

Norland, S., Wessel, R.C., \& Shover, N. (1981). Masculinity and delinquency. Criminology, 19, 421-433.

Parker, D., Harford, T., \& Rosenstock, I. (1994). Alcohol, other drugs, and sexual risk-taking among young adults. Journal of Substance Abuse, 6 (1), 87-93.

Patterson, G. (1996). Some characteristics of a developmental theory for earlyonset delinquency. In M. Lenzenweger \& J.J. Haugaard (eds), Frontiers of developmental psychopathology (81-124). New York: Oxford University Press.

Patterson, G., \& Yeorger, K. (1993). Developmental models for delinquent behavior. In S. Hodgins (ed.), Mental disorder and crime. Newbury Park, CA : Sage.

Reige, M.G. (1972). Parental affection and juvenile delinquency in girls. British Journal of Criminology, 12, 55-73.

Robins, L.N. (1986). The consequences of conduct disorder in girls. In D. Olweus, J. Block \& M. Radkey-Yarrow (eds), Development of antisocial and prosocial behavior (385-414). Orlando: Academic Press.

Rutter, M., Giller, H., \& Hagell, A. (1998). Antisocial behavior by young people. Cambridge, UK: Cambridge University Press.

Sampson, R., \& Laub, J. (1990). Crime and deviance over the life course: the salience of adult social bonds. American Sociological Review, 55, 609-627.

Sampson, R., \& Laub, J. (1993). Crime in the making: patbways and turning points through life. Cambridge, MA: Harvard University Press.

Sampson, R., \& Laub, J. (1997). A life-course theory of cumulative disadvantage and the stability of delinquency. In T.P. Thornberry (ed.), Developmental theories of crime and delinquency: advances in criminological theory, vol. 7. New Brunswick, NJ : Transaction Publishers.

Sampson, R., \& Laub, J. (2003). Life-course desisters? Trajectories of crime among delinquent boys followed to age 70. Criminology, 41, 555-592.

Sliverthorn, P., \& Frick, P. (1999). Developmental pathways to antisocial behavior: the delayed-onset pathway in girls. Development and Psychology, 11, 101-126.

Sliverthorn, P., Frick, P., \& Reynolds, R. (2001). Timing of onset and correlates of severe conduct problems in adjudicated girls and boys. Journal of Psychopathology and Behavioral Assessment, 23, 171-181. 
Simon, R. (1975). Women and crime. Lexington, MA: Lexington Books.

Smith, C., \& Brown, J. (1998). Sexual behaviors, extroversion, and alcohol use among college students. Journal of Alcobol \& Drug Education, 44 (1), 70-79.

Steffensmeier, D. (1980). Sex differences in patterns of adult crime, 19651977. A review and assessment. Social Forces, 57, 566-584.

Steffensmeier, D., \& Allen, E. (1988). Sex disparities in arrests by residence, race, and age: an assessment of the gender convergence/crime hypothesis. Justice Quarterly, 5, 53-80.

Steffensmeier, D., \& Streifel, C. (1993). Trends in female crime, 1960-1990. In C. Culliver (ed.), Female criminality: The state of the art (63-101). New York: Garland.

Stein, J.A., \& Newcomb, M. (1999). Adult outcomes of adolescent conventional and agentic orientations: a 20-year longitudinal study. Journal of Early Adolescence, 19 (1), 39-65.

Tracy, P., Wolfgang, M., \& Figlio, R. (1990). Delinquency in Two Birth Cohorts. New York: Plenum.

Wallace, J.M., Jr., Bachman, J.G., O’Malley, P.M., Schulenberg, J.E., Cooper, S.M., \& Johnston, L.D. (2003). Gender and ethnic differences in smoking, drinking, and illicit drug use among American 8th, 10th and 12th grade students, 1976-2000. Addiction, 98, 225-234.

West, D.J., \& Farrington, D.P. (1973). Who Becomes Delinquent? London: Heinemann.

West, D.J., \& Farrington, D.P. (1977). The Delinquent Way of Life. London: Heinemann.

Wolfgang, M., Figlio, R., \& Sellin, T. (1972). Delinquency in a birth cobort. Chicago: University of Chicago Press.

Wolfgang, M., Figlio, R., Tracy, P., \& Singer, S. (1985). The national survey of crime severity. U.S. Department of Justice. Washington, DC: Bureau of Justice Statistics. 


\section{ANNEXE 1}

Distribution des réponses aux items portant sur la délinquance à l'adolescence en fonction des quatre catégories de délinquantes identifiées par l'analyse de structure latente

\begin{tabular}{|c|c|c|c|c|}
\hline \multirow[b]{2}{*}{ Variables de base } & \multicolumn{4}{|c|}{ Catégories de délinquantes juvéniles } \\
\hline & $\begin{array}{c}I \\
\text { Conformisme }\end{array}$ & $\begin{array}{c}\text { II } \\
\text { Délits mineurs } \\
\text { avec violence }\end{array}$ & $\begin{array}{c}\text { III } \\
\text { Délits reliés } \\
\text { à la drogue }\end{array}$ & $\begin{array}{c}\text { IV } \\
\text { Délinquance } \\
\text { grave }\end{array}$ \\
\hline \multicolumn{5}{|l|}{ Violence mineure } \\
\hline non & 1,0000 & 0,0109 & 0,2718 & 0,0225 \\
\hline oui & 0,0000 & 0,9891 & 0,7282 & 0,9775 \\
\hline \multicolumn{5}{|l|}{ Violence majeure } \\
\hline non & 1,0000 & 0,6381 & 0,6251 & 0,1031 \\
\hline oui & 0,0000 & 0,3619 & 0,3749 & 0,8969 \\
\hline \multicolumn{5}{|l|}{$\begin{array}{l}\text { Crimes graves } \\
\text { contre la propriété }\end{array}$} \\
\hline non & 1,0000 & 0,9784 & 1,0000 & 0,5555 \\
\hline oui & 0,0000 & 0,0216 & 0,0000 & 0,4445 \\
\hline \multicolumn{5}{|l|}{$\begin{array}{l}\text { Crimes mineurs } \\
\text { contre la propriété }\end{array}$} \\
\hline non & 1,0000 & 0,7762 & 0,5962 & 0,0000 \\
\hline oui & 0,0000 & 0,2238 & 0,4038 & 1,0000 \\
\hline \multicolumn{5}{|l|}{ Vente de drogues } \\
\hline non & 1,0000 & 0,9998 & 0,8940 & 0,5198 \\
\hline oui & 0,0000 & 0,0002 & 0,1060 & 0,4802 \\
\hline \multicolumn{5}{|l|}{$\begin{array}{l}\text { Consommation } \\
\text { de drogues }\end{array}$} \\
\hline non & 1,0000 & 1,000 & 0,5127 & 0,3019 \\
\hline oui & 0,0000 & 0,000 & 0,4873 & 0,6981 \\
\hline $\begin{array}{c}\text { Probabilité } \\
\text { d'appartenance } \\
\text { à une catégorie }\end{array}$ & 0,221 & 0,514 & 0,155 & 0,110 \\
\hline $\begin{array}{l}\text { Nombre de } \\
\text { répondants } \\
(n=484)\end{array}$ & 107 & 249 & 75 & 53 \\
\hline $\begin{array}{l}\text { Khi-carré de } \\
\text { Pearson }\end{array}$ & \multicolumn{4}{|c|}{40,9874} \\
\hline $\begin{array}{c}\text { Khi-carré du rapport } \\
\text { de vraisemblance }\end{array}$ & \multicolumn{4}{|c|}{51,2382} \\
\hline Degrés de liberté & \multicolumn{4}{|c|}{45} \\
\hline
\end{tabular}


ANNEXE 2

Matrice de corrélation des modèles d'adolescents délinquants

\begin{tabular}{|c|c|c|c|c|c|c|c|c|}
\hline & 1 & 2 & 3 & 4 & 5 & 6 & 7 & 8 \\
\hline 1 & & 0,058 & $0,413 * * *$ & $0,241^{* * *}$ & $0,330 * * *$ & $-0,244^{* * *}$ & $-0,280 * * *$ & 0,057 \\
\hline 2 & 0,058 & & $0,451^{* * *}$ & $0,370 * * *$ & $0,239 * * *$ & $-0,072$ & 0,058 & $0,151 * * *$ \\
\hline 3 & $0,413^{* * *}$ & $0,451 * * *$ & & $0,586^{* * *}$ & $0,370 * * *$ & $-0,160 * * *$ & $-0,103^{*}$ & $0,096^{*}$ \\
\hline 4 & $0,241^{* * *}$ & $0,370 * * *$ & $0,586^{* * *}$ & & $0,355^{* * *}$ & $-0,130 * * *$ & 0,059 & $0,123^{* *}$ \\
\hline 5 & $0,330 * * *$ & $0,239 * * *$ & $0,370 * * *$ & $0,355^{* * *}$ & & $-0,171^{* * *}$ & $-0,064$ & $0,114^{* *}$ \\
\hline 6 & $-0,244 * * *$ & $-0,072$ & $-0,160 * * *$ & $-0,130$ ** & $-0,171 * * *$ & & $0,203 * * *$ & 0,048 \\
\hline 7 & $-0,280 * * *$ & 0,058 & $-0,103$ & 0,059 & $-0,064$ & $0,203 * * *$ & & $0,428 * * *$ \\
\hline 8 & 0,057 & $0,151^{* * *}$ & 0,096 & $0,123^{* *}$ & $0,114^{* *}$ & 0,048 & $0,428 * * *$ & \\
\hline
\end{tabular}

Notes: * $p<0,05, * * p<0,01, * * * p<0,001$

${ }^{1}$ Soutien parental au développement de l'identité en $1982,{ }^{2}$ Supervision parentale, ${ }^{3}$ Attachement à la famille, ${ }^{4}$ Engagement des parents envers l'école, ${ }^{5}$ Engagement à l'école, ${ }^{6}$ Pressions des pairs, ${ }^{7}$ Confiance aux pairs et ${ }^{8}$ Attachement aux pairs. 